\title{
Microstructural evolution induced mechanical property enhancement in cryogenically rolled Ce-modified SAF2507 super duplex stainless steel
}

Tian Zhou ${ }^{1}$, Yi Xiong ${ }^{1,2 *}$, Xiao-qin Zha ${ }^{3}$, Yan $\mathrm{Lu}^{1}$, Tian-tian $\mathrm{He}^{3}$, Feng-zhang Ren ${ }^{1,2}$, Ekta Rani ${ }^{4}$, Harishchandra Singh ${ }^{4}$, Jukka Kömi ${ }^{5}$, Marko Huttula ${ }^{1,4}$, Wei Cao ${ }^{4,6 *}$

${ }^{1}$ School of Materials Science and Engineering, Henan University of Science and Technology, Luoyang 471023, Henan, China

${ }^{2}$ Collaborative Innovation Center of Nonferrous Metals, Luoyang 471023, Henan, China

${ }^{3}$ Luoyang Ship Material Research Institute, Luoyang 471000, Henan, China

${ }^{4}$ Nano and Molecular Systems Research Unit, University of Oulu, FIN-90014, Finland

${ }^{5}$ Materials and Mechanical Engineering, Center for Advanced Steels Research, University of Oulu, FIN-90014, Finland

${ }^{6}$ School of Mechanical and Automotive Engineering, Anhui Polytechnic University, Wuhu 241000, Anhui, China

Abstract: Thermomechanical deformation is one of the most efficient and facile routes to tailor microstructure in structural materials for mechanical property enhancement. In this work, the Ce-modified SAF2507 super duplex stainless steels (Ce-SAF2507) were systematically subjected to various deformation levels of $30 \%-90 \%$ at cryogenic temperature $\left(-196{ }^{\circ} \mathrm{C}\right)$ to achieve superior mechanical performance. Cryogenic rolling rose fiber texture and induced ultra-fine grain refinement with size $\sim 10 \mathrm{~nm}$ in the selected steel. The high-density dislocations and deformation twins in the cryogenically rolled Ce-SAF2507 led to the nucleation and growth of martensite. An increase in the martensite volume fraction and the nanoscale grain refinement occurred at higher deformation levels. The higher hardness increment of austenite-martensite dual-phase compared to that of ferrite was due to the austenite-martensite's higher work hardening ability. Cryogenically rolled deformation resulted in the overall increase of the Ce-SAF2507 hardness. Further, the ultimate tensile strength and yield strength increased with the deformation level, but the elongation decreased with the same. Observed microstructural evolutions induced by cryogenic rolling enunciated the superiority of the same over conventional deformation methods to promote steel' mechanical properties.

Keywords: SAF2507 SDSS; Rare earth element Ce inclusion; Cryogenic rolling; Microstructural evolution; Deformation-induced martensitic transformation 


\section{Introduction}

Requirements for advanced materials' performance are escalating to save resources and tackle global demand for greenhouse gas reductions. In this regard, duplex stainless steel (DSS) has turned out a winner. The DSS is categorized in the high-alloyed steels with a biphasic microstructure simultaneously consisting of austenite and ferrite at an equivalent volume fraction. The presence of austenite phase reduces brittleness and grain growth tendency of high chromium ferrite. As a result, the fracture toughness is enhanced. This has also been demonstrated by Sieurin and Sandström [1], wherein the facture toughness of base and weld material of 2205 DSS was found to be more suitable for possible pressure vessel. It was found that the base material gave a very high fracture toughness at subzero temperature with ductile behavior. The chromium-rich ferrite, on the other hand, provides resistance to intergranular corrosion and stress corrosion in DSS. For instance, Adams et al. [2] performed a detailed investigation on the corrosion behavior of 2205 and 2507 DSS in the presence of organic acid/chloride environments and temperature effects. Better corrosion resistance was found for the 2507 DSS. The DSS owns a lower nickel content, but a higher pitting resistance equivalent numbers (PREN) compared to the austenitic counterparts. The high PREN and strength enable the DSS' applications in harsh industrial fields. As proposed by Nilsson and Wilson [3], the super duplex stainless steels (SDSS) are a subset of DSS with the PREN > 40. Among various 
available SDSS, SAF2507 represents the third generation DSS. Its excellent mechanical and anticorrosive properties make it widely used in petrochemical, papermaking, wastewater treatment, and other tough industrial fields.

To date, the mechanical deformation of the SAF2507 SDSS are mainly performed at high temperatures. Such hot rolling deformation changes microstructures of the SAF2507 SDSS and varies its properties to meet users' requirement. Mishra et al. [4] performed isothermal hot compression tests on DSS to study its microstructural evolution. The dynamic recovery and continuous dynamic recrystallization were found in the ferrite phase while the discontinuous dynamic recrystallization occurred in the austenite. Hot torsion tests on DSS was also reported by Duprez et al. [5]. The yielded mechanical behavior was found dependent on the dynamic recovery of the ferrite phase and ductility of DSS correlated with the amount of dynamic softening following the austenite recrystallization. As a result, the significant microstructure modifications and corresponding dynamic softening mechanism of SAF2507 SDSS have systematically been studied under hot processing. However, heat treatments of DSS can also deteriorate its properties due to the appearance of unwanted phases at elevated temperatures. As reported by Hosseini et al. [6], precipitation of the $\sigma$ phase with different morphology (blocky and fine coral shape) was found to occur between 630 and $1010{ }^{\circ} \mathrm{C}$ during hot deformation. Pitting corrosion testing along with thermodynamic calculations showed the initiation of corrosion near the fine coral shaped $\sigma$ phase in heat treated DSS. Further, complications also arise from the difference in the hot deformation behavior of two phases and elude microstructure 
evolution. As a mature processing technology, facile room temperature cold deformation has come out as the effective steel processing technology with characteristics of high dimensional accuracy and stable structure. Breda et al. [7] studied cold rolled SAF2205 DSS to probe the strain induced martensite in the DSS. Despite the presence of austenite phase with low stacking fault energy, the DSS was found more stable than AISI 304L. Further, the extent of martensitic transformation was found significantly lower than in the metastable austenite stainless steel. The Ce modified SAF2507 was cold rolled in a latest work of Zhou et al. [8]. Along with the formation of strain induced martensite phase, increase in the strength index and decrease in the plasticity index were observed. Despite these progresses, dynamic recovery is bound to happen in the deformed microstructure during the room temperature cold rolling. This inevitably weakens the work hardening and strengthening effect. An alternative deformation route was referred at cryogenic temperatures such as the work reported by Xiong et al. [9]. A higher yield strength and ultimate tensile strength were noted in cryogenically rolled austenite stainless steel compared to the cold rolled ones. This enhancement was attributed to the effective suppression of the dynamic recovery at cryogenic temperatures.

Compared to scarcely reported cold rolling of SAF2507 (Zhou et al. [8]), the cryogenically rolled deformation of the SDSS has not been seen in the literature, to the best of our knowledge. Aiming to a cost-effective processing and superior thermal processing performances, Ce-modified SAF2507 SDSS (Ce-SAF2507) with an increased $\mathrm{N}$ content are selected in the present study. The key improvement of 
Ce-SAF2507 is to reduce the consumption of precious element $\mathrm{Ni}$ by increasing the content of comparatively cheaper $\mathrm{N}$ element appropriately. Further, the inclusion of rare earth element $\mathrm{Ce}$ has turned out to yield optimized microstructure and superior mechanical performances as reported in the work of Zhou et al. [8]. In the present work, Ce-SAF2507 has been subjected to cryogenic rolling under the deformation level in the range of $30 \%-90 \%$. Microstructural evolution such as ultra-fine grains, high austenite to martensite transformation and suppressed dynamic recovery have been noticed. Thanks to the microstructural evolution, the mechanical properties have been substantially enhanced. The present microstructure and mechanical property evolution are hoped to serve support and test guidance for the industrial application of SDSS in various fields such as in cold and corrosive environments.

\section{Experimental procedures}

Components of the Ce-SAF2507 were measured (in wt $\%$ ) as a balance $\mathrm{Fe}$ matrix with $0.023 \mathrm{C}, 0.35 \mathrm{Si}, 0.8 \mathrm{Mn}, 25 \mathrm{Cr}, 5.4 \mathrm{Ni}, 3.4 \mathrm{Mo}, 0.5 \mathrm{~N}$, and $0.08 \mathrm{Ce}$. In order to obtain qualified plates, Ce-SAF2507 SDSS ingot was forged and hot rolled. The plates were cut into $100 \times 30 \times 5 \mathrm{~mm}^{3}$ sheet specimens and divided into 5 groups for the preparation. All specimens underwent a solution treatment at $1050{ }^{\circ} \mathrm{C}$ for $1 \mathrm{~h}$, and subsequently, a water quenching to room temperature. Four groups of specimens were cryogenically rolled, with the deformation level of $30 \%, 50 \%, 70 \%$ and $90 \%$. It should be noted that the specimens need to be soaked in liquid nitrogen ( 10-15 min) prior to each rolling pass in order to cool the specimens homogenously. The rolling speed was fixed to $0.2 \mathrm{~m} / \mathrm{s}$ and rolling reduction to $5 \%$ during the cryogenic rolling 
(without any lubricant).

Microstructure measurements were carried out via ZEISS Z1M optical microscopy (OM) and JEM-2010 transmission electron microscopy (TEM). To prepare samples for $\mathrm{OM}$, specimens were etched for $3 \mathrm{~s}$ in aqua regia $\left(1 \mathrm{HNO}_{3}: 3 \mathrm{HCl}\right)$ on the longitudinal sections. The TEM samples were ion-beam thinned from the $3 \mathrm{~mm}$ disks punched from the rolling plane of the specimens. The phase fraction and strain distribution analysis were carried out from the X-ray diffraction (XRD) patterns of samples examined by Bruker D8 Advance X-ray diffractometer. This experiment was performed in the theta-2theta step scan mode with a step size of $0.02^{\circ}$ and angular range $40^{\circ}-100^{\circ}$ utilizing $\mathrm{Cu} \mathrm{K} \alpha$ source. Subsequently, the hardness of the two phases under different deformations was measured by NHT2 nanoindentor. During the nano-indentation, the load was kept at $100 \mathrm{mN}$, and the holding time was $10 \mathrm{~s}$. In order to obtain an accurate hardness value, a minimum of 20 indentations was performed for each phase. Meanwhile, the overall Vickers hardness of Ce-SAF2507 was also measured by MH-3 Vickers microhardness tester under a $100 \mathrm{~g}$ weight load and 10s loading time. By employing the above methods, the microstructural evolution of cryogenically rolled Ce-SAF2507 and its corroboration with the mechanical property were carried out systematically.

The austenite volume fraction of Ce-SAF2507 before and after cryogenic rolling can be quantitatively obtained as follow. De et al. [10] performed detailed XRD studies to quantify the deformation induced martensite phase and derived the following equation, 


$$
V_{\gamma}=\frac{\frac{1}{n} \sum_{\mathrm{j}=1}^{n} \frac{I_{i}^{j}}{R_{i}^{j}}}{\frac{1}{n} \sum_{\mathrm{j}=1}^{n} \frac{I_{\gamma}^{j}}{R_{\gamma}^{j}}+\frac{1}{n} \sum_{\mathrm{j}=1}^{n} \frac{I_{\alpha}^{j}}{R_{\alpha}^{j}}}
$$

wherein, $\mathrm{n}, \mathrm{I}$ and $\mathrm{R}$ are defined as peak number of XRD peaks in a particular phase, the integral intensity from the reflecting plane, and the scattering factor of the material, respectively. The same equation has also been implemented here in this work to calculate the phase fraction.

Williamson-Hall (W-H) method is used to estimate microstrain variation following the cryogenic rolling process. As per $\mathrm{W}-\mathrm{H}$ method, the diffraction peak broadenings are described as [11]:

$$
\begin{aligned}
& \left(\sigma_{m}\right)^{2}=\left(\sigma_{\text {in }}\right)^{2}+\left(\sigma_{\text {strain }}\right)^{2}+\left(\sigma_{\text {size }}\right)^{2} \\
& \sigma^{2}=\left(\sigma_{m}\right)^{2}-\left(\sigma_{\text {in }}\right)^{2} \\
& \sigma^{2}=(2 \varepsilon \tan \theta)^{2}+\left(\frac{0.9 \lambda}{l \cos \theta}\right)^{2} \\
& \sigma^{2} \cos ^{2} \theta=4 \varepsilon^{2} \sin ^{2} \theta+\left(\frac{0.9 \lambda}{l \cos \theta}\right)^{2}
\end{aligned}
$$

In these equations (i.e. Eqs. 2-5), $\sigma$ is the FWHM of the Bragg peak (in radian), $2 \theta$ the Bragg angles, $\lambda$ the wavelength of the X-ray source $(1.5406 \AA), l$ the average crystallite size in $\mathrm{nm}$, and $\varepsilon$ the microstrain. Here, $\sigma$ in counts for the instrumental broadening. It can be retrieved from the diffraction broadening of the reference sample (original solid solution sample) for further W-H analysis. The slope of the fitted line obtained from the linear plot of $\sigma^{2} \cos ^{2} \theta v s$. $4 \sin ^{2} \theta$ (Eqs. 2-5) gives $\varepsilon^{2}$.

\section{Results}

\subsection{Metallographic structure}


Microstructure analysis of cryogenically rolled Ce-SAF2507 has been carried to shed light on correlations between microstructure evolution and mechanical behaviors. Fig. 1 shows the microstructure evolution of pre- and post-cryogenically rolled Ce-SAF2507. As mentioned in the experimental section, the specimens are etched before carrying out the OM measurements. Due to the corrosion resistance differences in austenite and ferrite phase, the ferrite is etched profoundly into a dark phase, whereas lesser etching of the austenite phase led to a brighter region. As-cast Ce-SAF2507 microstructure is shown in Fig. 1(a) and 1(b). Numerous casting flaws are also expected to exist, resulting in the poor mechanical performance [12]. A specimen submitted to solutioning heat treatment shows the austenite phase is evenly distributed in the ferrite matrix (Fig. 1(c)). Compared with the as-cast microstructure, the casting flaws are vanished, and the grain size is significantly reduced in the solid solution state.

Post $30 \%$ deformation, the austenite grains were significantly elongated along the rolling direction and uniformly distributed (Fig. 1(d)). With the further increase in deformation level to $50 \%$, the deformation degree of ferrite and austenite increases. Rod-like shape grains were formed in austenite and ferrite phases with increased aspect (length to width) ratio (Fig. 1(e)). At a deformation level of $70 \%$ (Fig. 1(g)), the ferrite and austenite grains intercalate with each other in bands due to the continuous effect of stress. The resulting deformation also induces the austenite-martensite transformation, further reducing the bandwidth and leading to denser interlacing of the two phases. As the deformation level reaches to $90 \%$, Fig. 
1(i), the grains are stretched into interwoven slender spicules, indicating that the microstructure is transformed into a fibrous texture. Similar observation of wavy banded grains have also been reported in cold rolled DSS by Ryś and Cempura [13], which occur due to strain localization in biphasic materials and act as strong obstacles for the dislocation motions. In general, the wider band grains are mainly induced by the normal stress, leading to the continual decrease in the bandwidth. On the contrary, the narrower band grains are susceptible to shear stress. Thus, they are curved in a wavy shape (Fig. 1(i)).
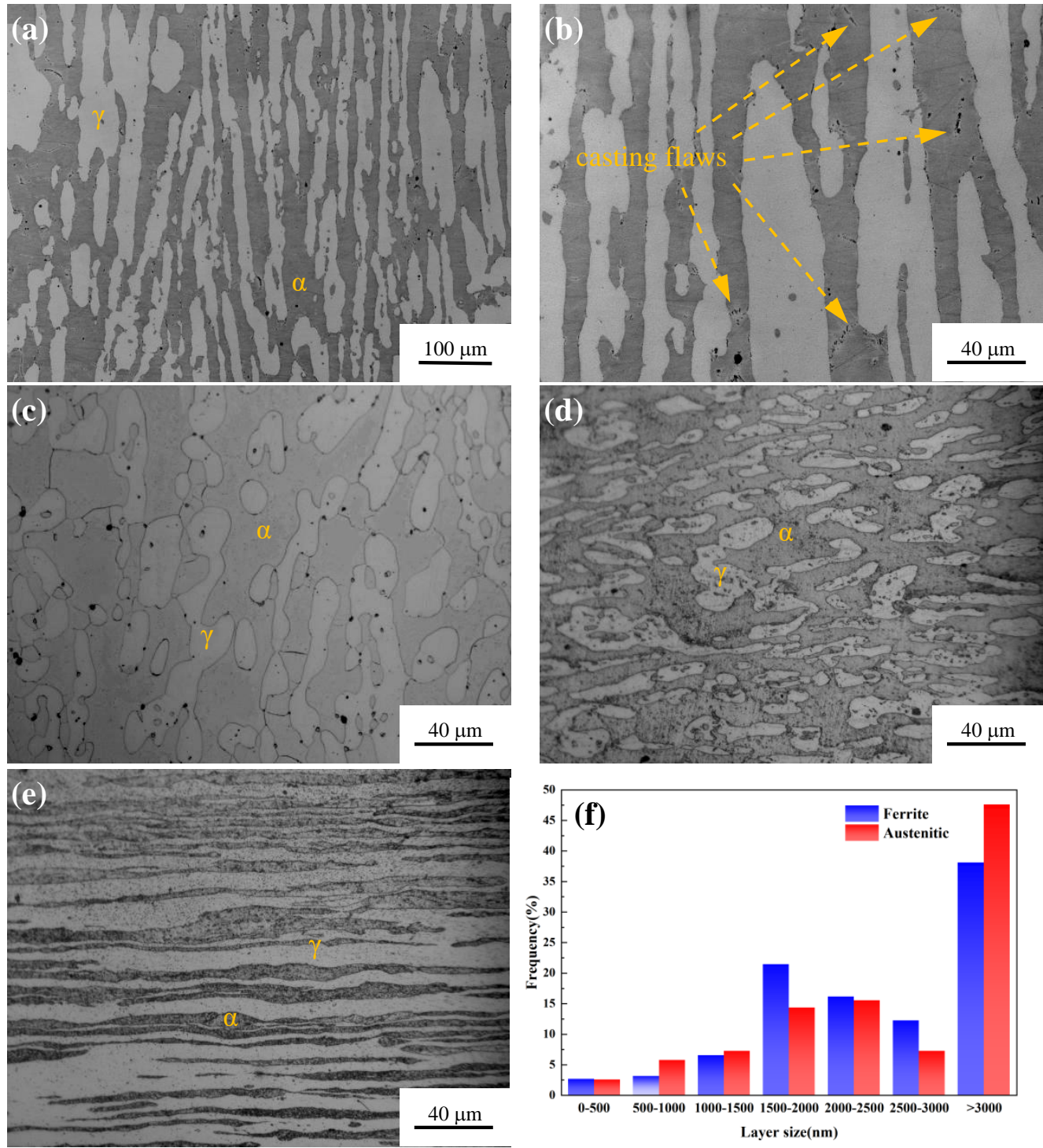

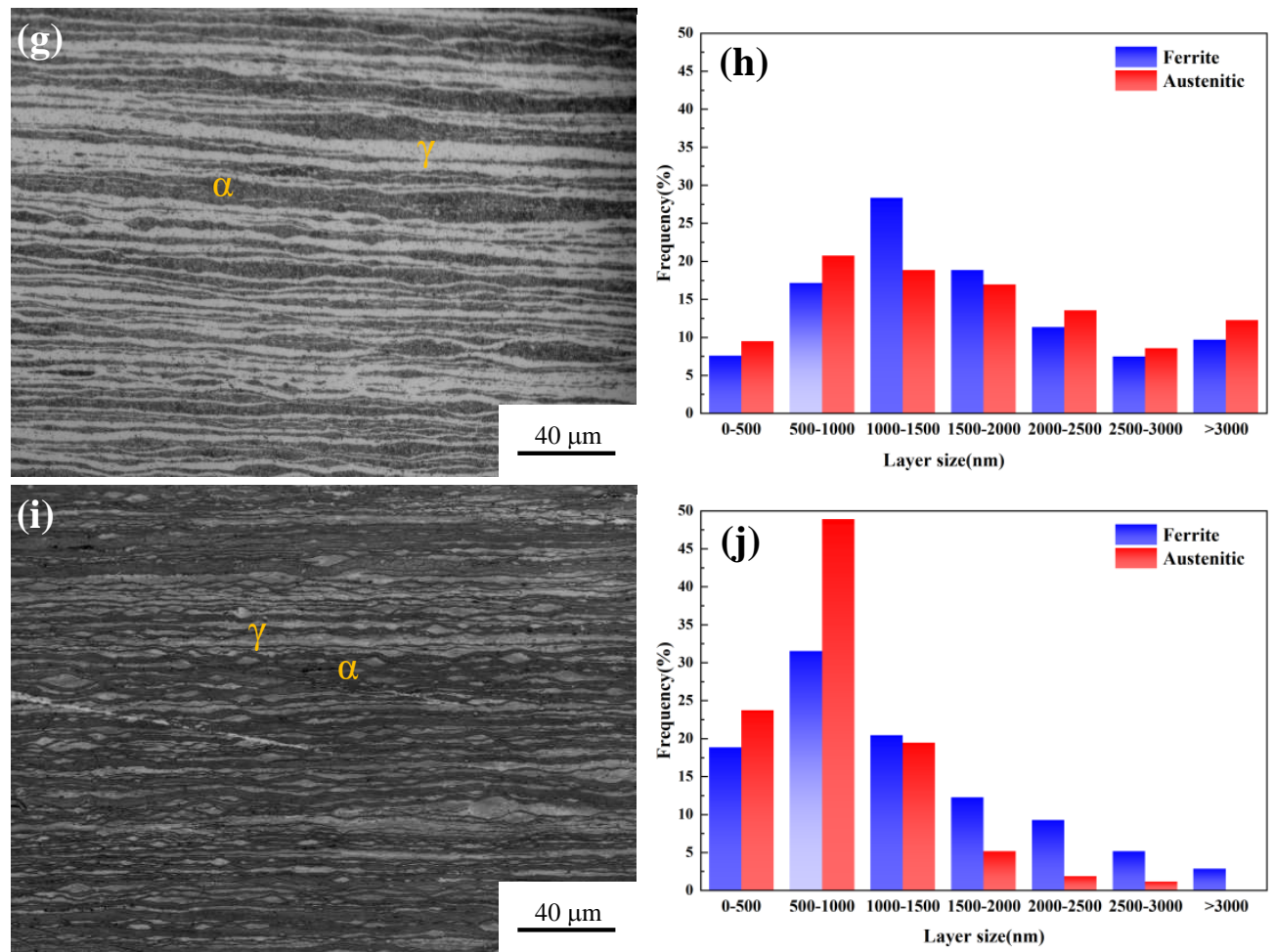

Fig. 1 Optical micrographs of Ce-SAF2507 in as-cast condition (a,b), solid solution state (c), and cryogenically rolled by $30 \%$ (d). Optical micrographs and corresponding frequency histogram of rolled Ce-SAF2507 by $50 \%(e, f), 70 \%$ $(\mathrm{g}, \mathrm{h})$ and $90 \%(\mathrm{i}, \mathrm{j})$.

A comparison of the distribution of deformed grain bandwidth of the two phases in the range of $50 \%-90 \%$ deformation level shows that the grain bandwidth of the two phases is generally more than $3 \mu \mathrm{m}$ at $50 \%$ deformation. Whereas, elevation of the deformation level to $90 \%$ leads to a gradual decrease in grain bandwidth down to $<1 \mu \mathrm{m}$. Under a high deformation level, the grain refining effect of austenite is more evident than that of ferrite. This refinement is due to the deformation-induced martensitic transformation, which significantly reduces the bandwidth and dramatically enhances the grain refinement.

\subsection{TEM observations}

Fig. 2 depicts the observed TEM images of cryogenically rolled Ce-SAF2507. At 
a strain level of $30 \%$ (Fig. 2(a, b)), the deformed microstructure of Ce-SAF2507 shows the presence of large number of interleaved twins in the austenite phase with a low stacking fault energy (SFE) (Fig. 2(a)). The nucleation sites are provided for deformation-induced martensite therein. Due to the high SFE, ferrite does not support the twin formation (Fig. 2(b)). Whereas the dislocation groups in the ferrite phase were formed from the intertwined high-density dislocations. When the deformation was increased to $90 \%$ (Fig. 2(c-f)), the austenite is found to be interwoven with the ferrite, blurring the grain boundary. In addition to the formation of twins, one can also notice the presence of ladder-like Moiré fringes (Fig. 2(d)). The formation of Moiré fringes is attributed to an accumulation of high-density stacking faults during the deformation process $[14,15]$. During the rolling process, extensive plastic deformation provides enough driving force for deformation-induced martensitic transformation. Increase in the volume fraction of deformation-induced martensite due to the interactions between dislocations and deformation twins further happens. A large amount of martensite grows as nucleation in the austenite, as shown in the bright (Fig. 2(e)) and dark (Fig. 2(f)) field images. On the other hand, in the ferrite phase, dislocations move mainly by slip and cross slip. The interaction between dislocations results in the formation of many substructures in the ferrite, which results in the significant fragmentation of ferrite grains (Fig. 2(g)). Fig. 2(h) shows the dark field image of $90 \%$ deformed Ce-SAF2507 indicating the ferrite grain breakage. Selected area electron diffraction pattern presents a ring structure, indicating that the grain has significantly been refined to the nanoscale $[10,12]$, even up to about $10 \mathrm{~nm}$. 

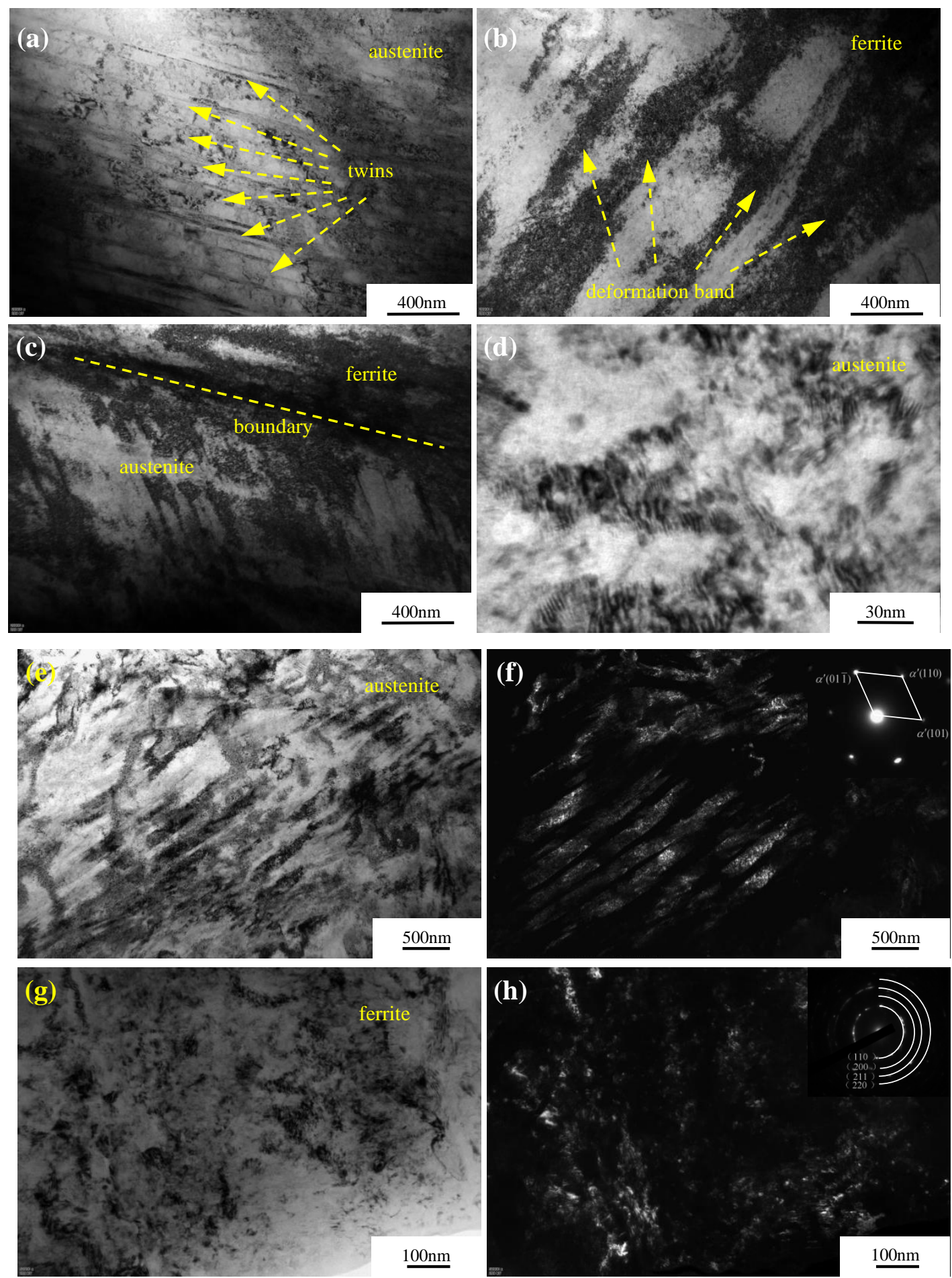

Fig. 2 Observed microstructure of Ce-SAF2507 SDSS cryogenic rolled using TEM, (a, b) cryogenic rolled by $30 \%$, and (c-h) cryogenic rolled by $90 \%$. 


\subsection{Macrostructural changes: XRD evolutions}

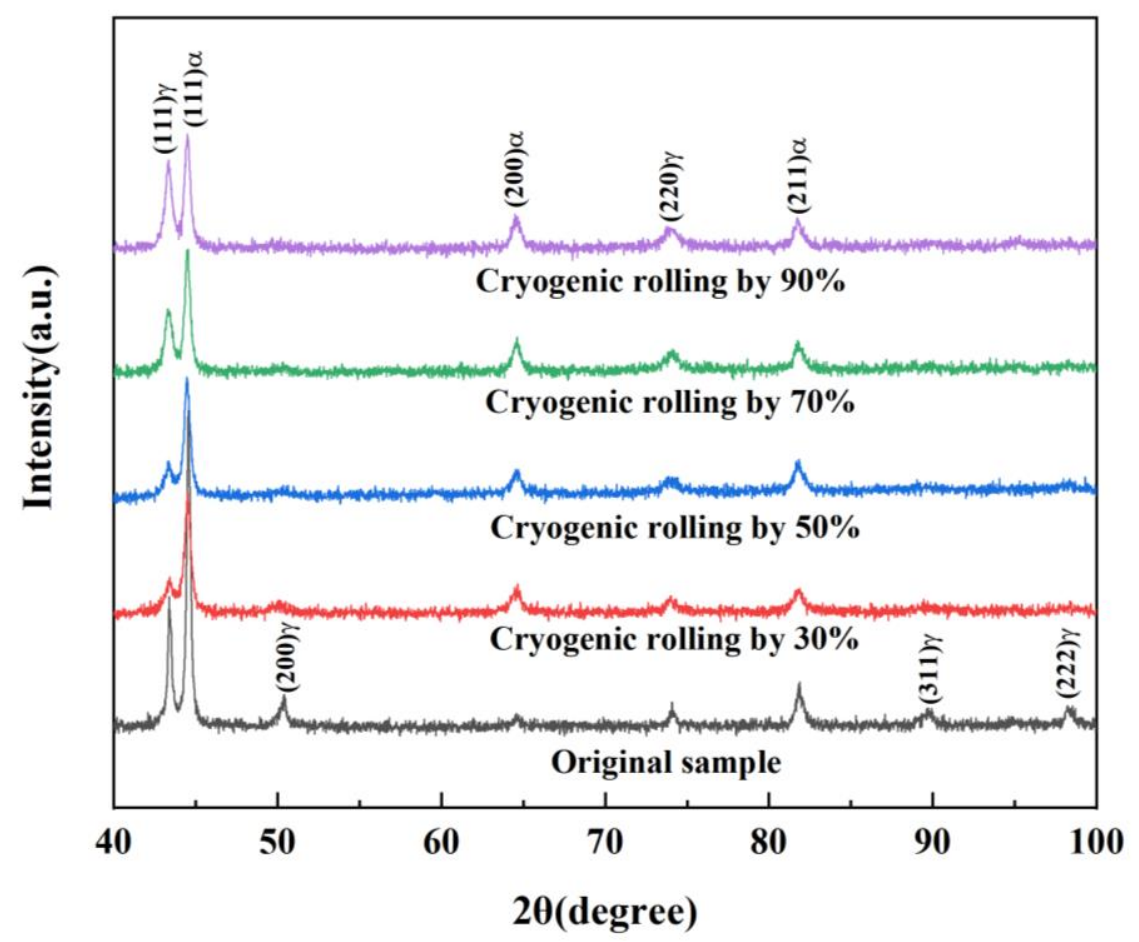

Fig. 3 X-ray diffraction patterns of cryogenically rolled Ce-SAF2507 at different deformation levels.

The XRD patterns of cryogenically rolled Ce-SAF2507 are presented in Fig. 3. Diffraction pattern of solid solution mainly shows $\alpha$ (111), $\alpha$ (200), and $\alpha$ (211) diffraction peaks corresponding to ferrite and $\gamma(111), \gamma(200), \gamma(220), \gamma(311)$, and $\gamma$ (222) peaks corresponding to the austenite phase [16,17]. Intensities of peaks corresponding to austenite phase gradually decrease with the deformation level. Further, few peaks, such as $\gamma(311), \gamma(200)$, and $\gamma(222)$, disappeared completely. This observation is inferred to the stress-induced phase transformation from austenite to martensite during the deformation process. The increase in the deformation level leads to the increase in the volume fraction of deformation-induced martensite and the corresponding decrease of the austenite. According to formula (1), the austenite and 
ferrite volume fraction of solid solution Ce-SAF2507 is $51.6 \%$ and $48.4 \%$, respectively. Whereas, at $90 \%$ deformation, the volume fraction of austenite decreases to $8.2 \%$. This missing austenite is replaced by deformation-induced martensite with a volume fraction of $43.4 \%$ and a relative transformation rate of $84.1 \%$. However, even at higher level of deformation (90\%), the austenite phase was not able to reach an entire martensitic transformation. The austenite to martensite transformation of Ce-SAF2507 at different deformation levels is shown in Fig. 4.

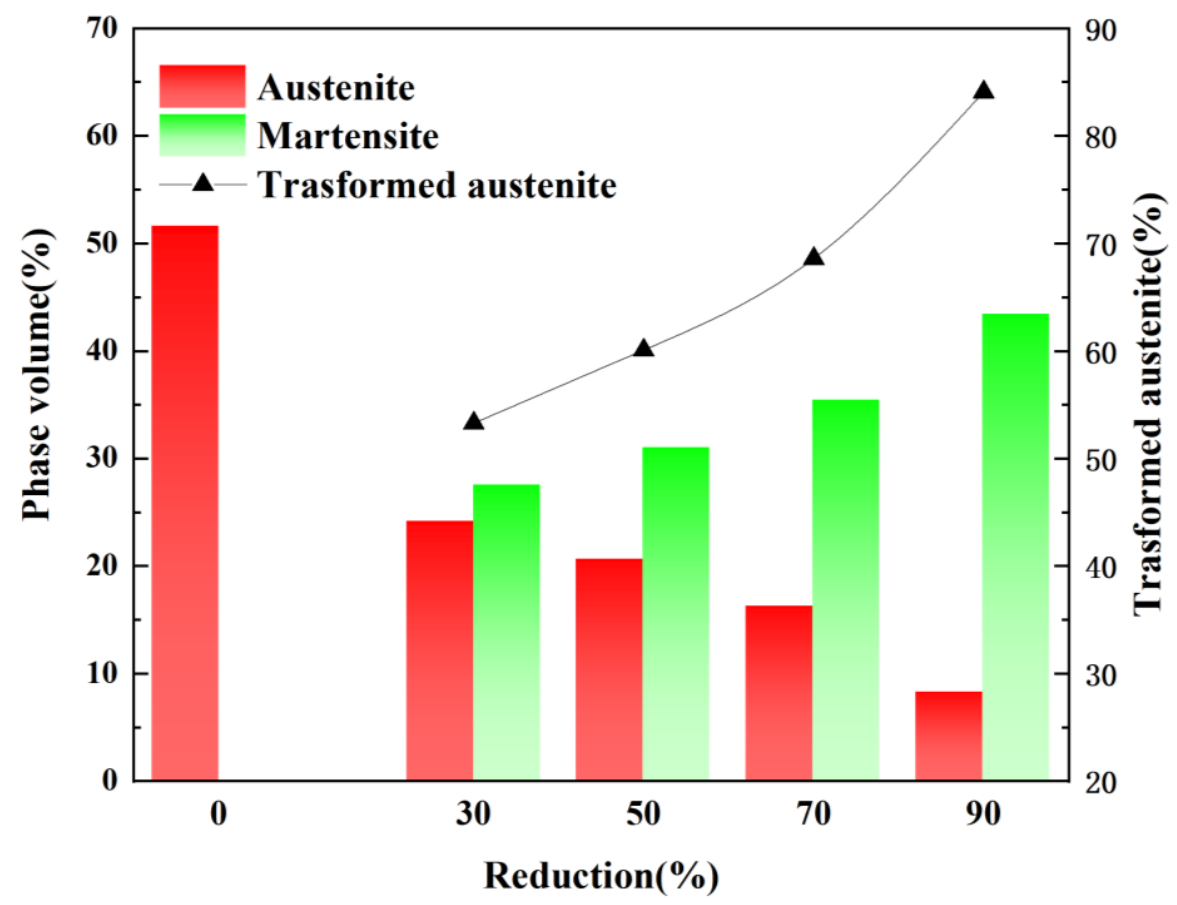

Fig. 4 Martensitic transformation of cryogenically rolled Ce-SAF2507 at different deformation levels.

\subsection{Strain analysis}

Apart from the observed changes in the intensity of diffraction peaks, change in the full width at half maximum (FWHM) has also been noticed and therefore analyzed here. This change in FWHM for cryogenically rolled Ce-SAF2507 can be correlated with the microstrain generated in Ce-SAF2507. Microstrain is known as the 
local distortion in the crystal lattice, such as dislocations in ferrite or twins in the austenite phase. It introduces variations in the lattice parameters and also leads to higher FWHM of the diffraction peaks $[18,19]$. Utilizing Eqs. 2-5, the calculated microstrain is plotted in Fig. 5 as a function of various deformation level. As noted, a higher level of strain is observed for austenite phase compared to ferrite, indicating its higher work hardening ability. During the deformation process, the metastable austenitic phase is transformed into the martensitic phase, resulting in the higher concentration of strain in the austenite. The strain increases with the deformation level for both phases. The strain increment of austenite is higher than that of ferrite, which is further corroborated with the observed microhardness.

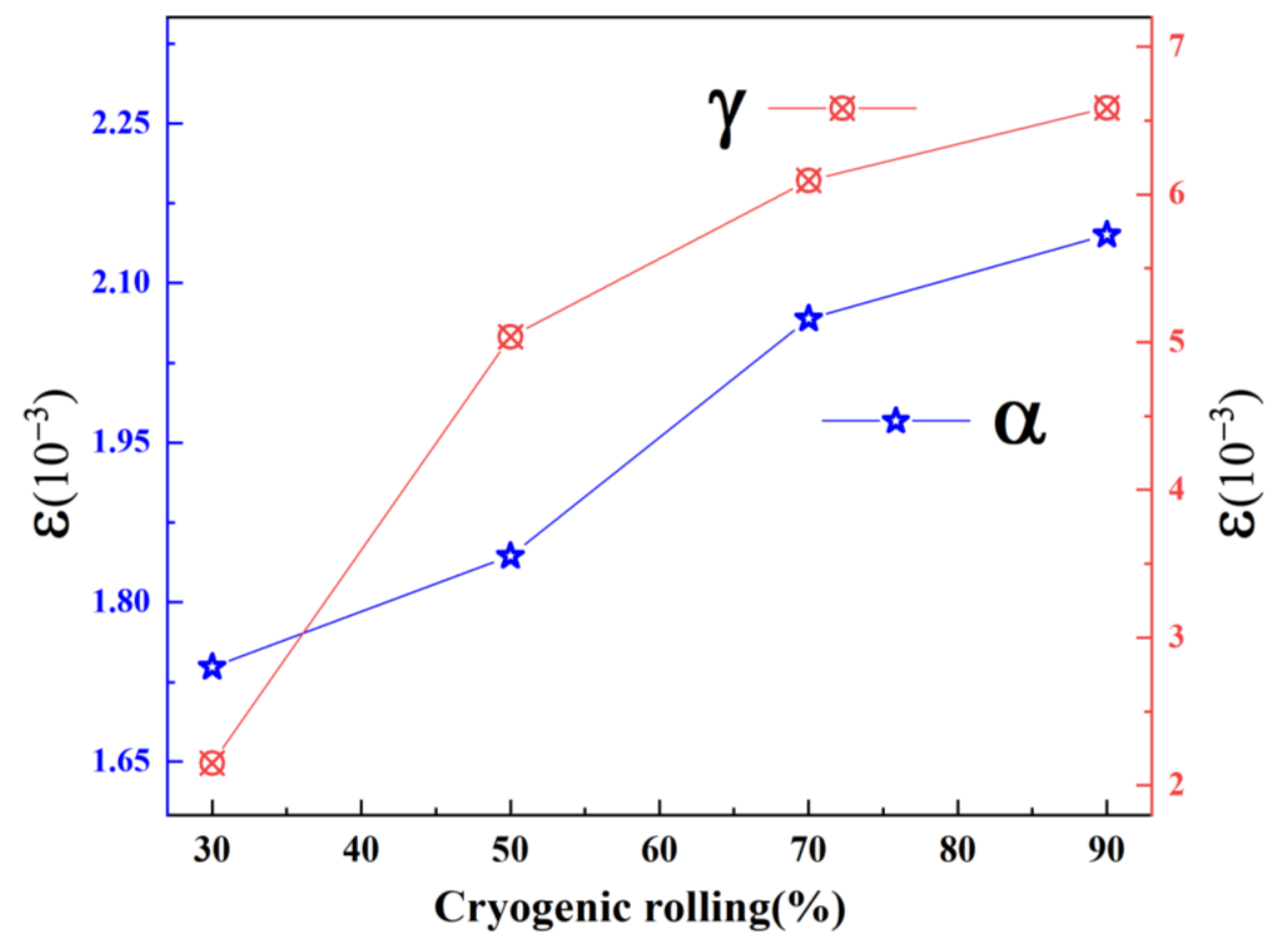

Fig. 5 Calculated microstrain in the ferrite and austenite phases in cryogenically rolled Ce-SAF2507 at different deformation levels.

\subsection{Mechanical properties}


The influence of deformation level on the microhardness of Ce-SAF2507 is depicted in Fig. 6. In the solid solution Ce-SAF2507, the nano-indentation hardness of ferrite and austenite comes out to be $4.516 \mathrm{GPa}$ and $5.237 \mathrm{GPa}$, respectively, and the overall Vickers hardness is HV 374. The hardness of both austenite and ferrite tends to increase with the deformation level. When the deformation level reaches $90 \%$, the nano-indentation hardness of ferrite and austenite increased to $5.805 \mathrm{GPa}$ and 7.612 GPa, respectively, and the overall Vickers hardness increased to HV 593. Thus, in the process of cryogenic rolling, the overall microhardness of Ce-SAF2507 increases gradually. Comparison of Fig. 5 and Fig. 6 shows a significantly larger amount of strain and the hardness increment in the austenite, which indicates the higher work hardening ability of austenite. Comparing Fig. 4 and Fig. 6, an increase in the martensite volume fraction comes along with the hardness increase of the austenite phase in Ce-SAF2507. Ran et al. [20] studied the microstructural evolution of cold rolled 19Cr DSS and found that, the microhardness of materials is mainly affected by dislocation slip in ferrite phase and dislocation slip in the initial stages of deformation followed by mechanical twining, and martensite phase formation at higher deformation levels. During the deformation process, the dislocation increment and accumulation mainly occurs in the ferrite. Many dislocation cells or subgrain boundaries are formed due to the continuous entanglement of dislocations, which impedes dislocation motion and further refines ferrite grains to the nanometer level, thus increasing the hardness of the material. While in the low SFE austenite, a large number of twins are seen to be formed. Simultaneously, deformation-induced 
martensite continues to nucleate and grow at the defects, which combines twins to anchor the dislocations effectively and hinder dislocation motion. The effect of lattice distortion caused by austenitic transformation is higher than that caused by ferrite grain refinement, which is also supported by the higher strain level of austenitic phase during the deformation of Ce-SAF2507, as shown in Fig. 5. This interpretation is further backed by Papula et al. [21], wherein the authors investigated the strain hardening of cold rolled lean-alloyed DSS. Their nanoindentation results showed that the susceptibility of work hardening increases with the decrease in SFE, thus, explaining the higher work hardening ability of austenite compared to the ferrite phase. These two different microstructure evolutions of the two phases contribute significantly to the overall increase of the hardness.

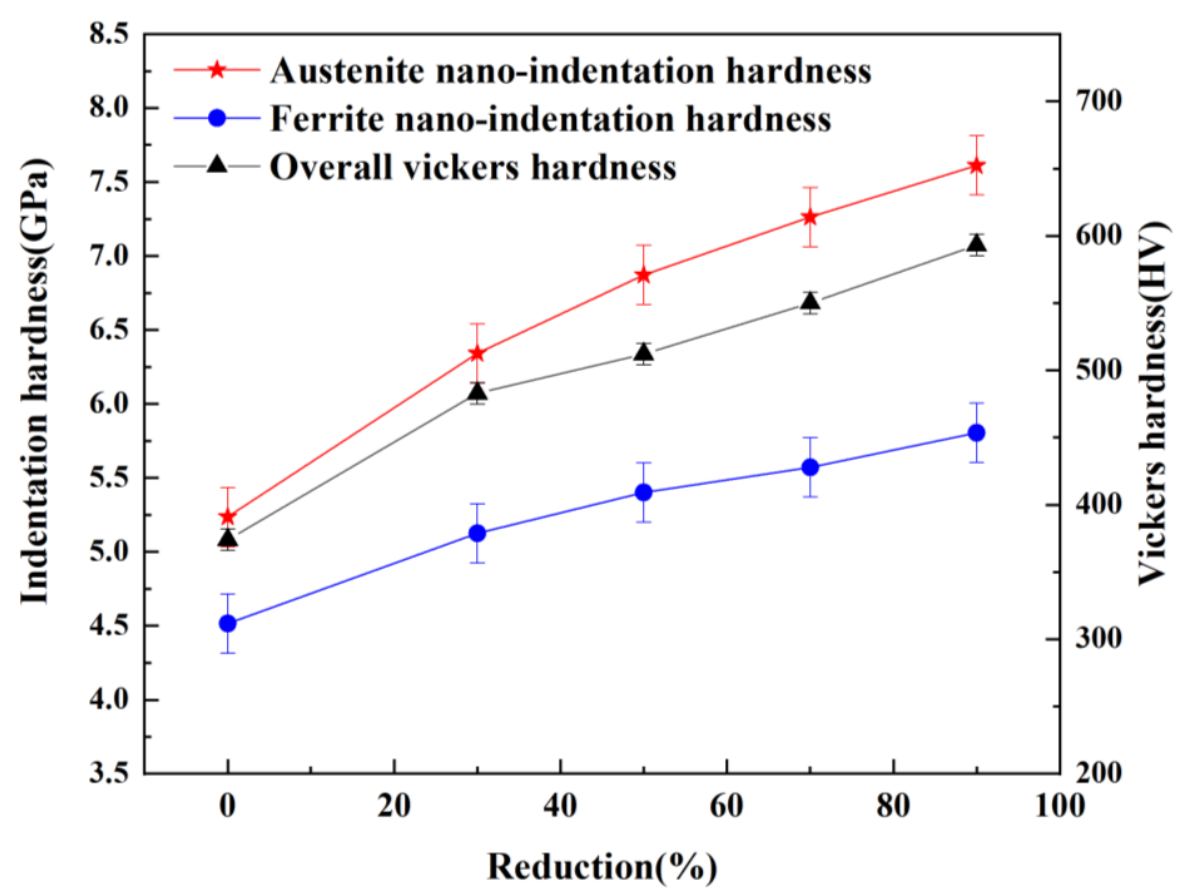

Fig. 6 Influence of deformation level on the microhardness of Ce-SAF2507.

Fig. 7 depicts the influence of deformation level on the tensile properties of 
Ce-SAF2507. The ultimate tensile strength, yield strength and elongation of Ce-SAF2507 in the solid solution state are observed to be $781 \mathrm{MPa}, 578 \mathrm{MPa}$ and $15.2 \%$, respectively. With the increase in the deformation level, the strength increases continuously but the plasticity decreases. As the deformation level reaches $90 \%$, the ultimate tensile strength and yield strength increase to $1682 \mathrm{MPa}$ and $1641 \mathrm{MPa}$, respectively, while the elongation decreases to $2.9 \%$. This phenomenon has been attributed to the joint action of working hardening, grain refinement and deformation-induced martensitic transformation. This is found to be consistent with the previous studies (Zhou et al. [8]) on strengthening mechanism of cold rolling at room temperature. Moreover, the strength evolution law confirms the microhardness test results as well. 


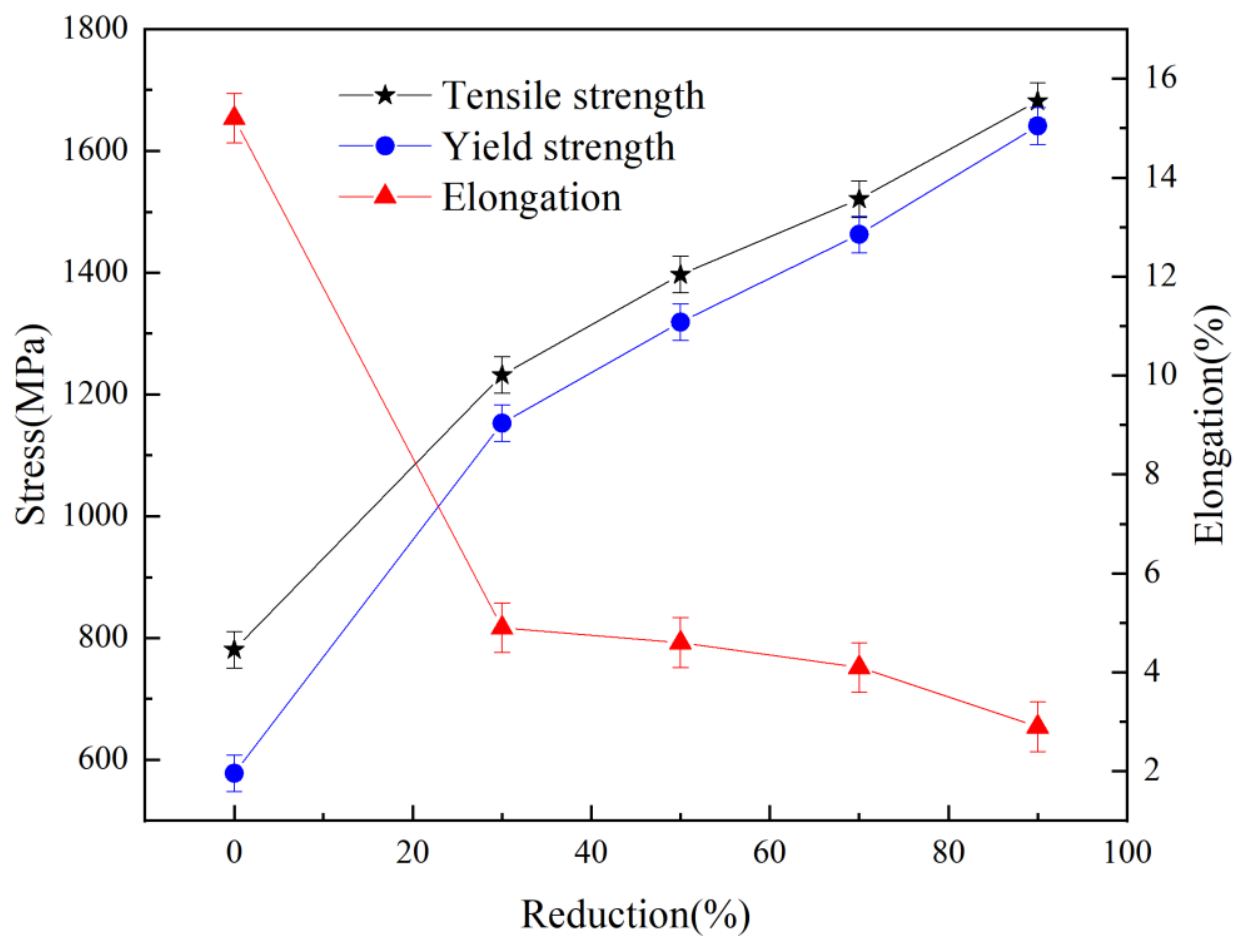

Fig. 7 Influence of deformation level on the tensile properties of Ce-SAF2507.

\section{Discussion}

Numerous aforesaid interesting findings of cryogenically rolled Ce-SAF2507 over previously reported cold and hot rolled can be summarized in many aspects. During the deformation process of cryogenic rolling, displacements accumulate and rearrange to form the sub-structure in the ferrite phase of Ce-SAF2507, thus refining the grain size to the nanometer level. At $90 \%$ deformation level, fine ferrite grains can eventually reach to about $10 \mathrm{~nm}$. Refinement of grain size has also been noted in austenite-martensite dual-phase. Observed ultra-fine grains (Fig. 1) are highly essential for high-strength steel when used in automobiles for better fuel efficiency. Grain refinement down to nanoscale is further considered to be one of the critical 
parameters affecting the mechanical properties positively.

In the austenite phase, the presence of a substantial number of twins existed in cryogenically rolled Ce-SAF2507, and the interactions between those twins provide nucleation sites for deformation-induced martensite at $30 \%$ deformation (Fig. 4). Further, an increase in the deformation level to $90 \%$ leads to an increase in the amount of martensite (Fig. 4). Various deformation modes can initiate the deformation-induced martensitic transformation. Shrinivas et al. [22] investigated the deformation induced martensitic formation in cold rolled 304 and 316 stainless steel and attributed shear band intersections to the preferential nucleation sites for martensite. The other possible nucleation sites also include deformation twins, stacking faults, and lath martensite. This transformation process is thus influenced by various factors, including stress level, strain rate, and deformation temperature.

Free energy difference between the newly formed martensite and the parent austenite also drives the martensitic transformation. It is well known that strain-induced austenite-martensite transformation takes place through the nucleation and growth process. This transformation occurs when the deformation-induced mechanical along with the chemical driving force exceeds the chemical driving force of phase transition. In the present case, the austenite-martensite transformation occurs at a cryogenic temperature, which is far below the initiation temperature of martensite. This suggests that the transformation is possible only because of the significant suppression of dynamic recovery at the cryogenic temperature [23-25]. Thus, during the process of deformation, large amounts of dislocations and stacking faults are 
formed followed by the nucleation and growth of strain-induced martensite. Further, the large strain applied during various levels of deformation leads to the continuous generation of twins followed by interaction between different orientation twins, which provides a large number of locations for the nucleation and continuous growth of martensite [26,27]. Therefore, the higher deformation level is associated with the larger volume fraction of strain-induced martensite.

Cryogenic rolling has been found superior in deformation-induced martensitic transformation over the room temperature cold rolling. Previous studies (Zhou et al. [8]) shows the martensitic transformation rate reached only $71.3 \%$ under $90 \%$ deformation at room temperature. Whereas, cryogenic rolling can reduce austenite to less than $10 \%$ leading to higher martensite volume fraction and significantly improves the strength and hardness to meet higher industrial production needs. This betterment is because of the higher work hardening ability of martensite, which significantly reduces the crack propagation rate. This observation is further supported by a prior art of Xiong et al. [9] towards the effect of room temperature cold rolling and cryogenic rolling on the microstructure and properties of austenitic stainless steel. Both room temperature and cryogenic rolling led to a remarkable enhancement in the hardness. However, it was found to be higher for cryogenic deformation, which was attributed to the observed higher volume fraction of martensite at same strain rate.

Further, comparison of strain analysis for cold (Zhou et al. [8]) and cryogenically rolled Ce-SAF2507 shows similar trends, i.e., increase in strain with the increase in deformation level. However, it is important to mention here that the strain 
accumulation of ferrite is lower $\left(20 \times 10^{-4}\right)$ than that of the room temperature cold rolling $\left(52 \times 10^{-4}\right)$, which is rather intriguing. Whereas, the strain accumulation of austenite is significantly higher $\left(70 \times 10^{-4}\right)$ than that of room temperature cold-rolled austenite $\left(48 \times 10^{-4}\right)$ and ferrite (cold and cryogenically rolled). Observed higher strain accumulation of austenite phase can be understood due to the formation of the strain-induced martensitic transformation and nano-grains, which further explains the observed trends in the nanoindentation and hardness. Observed lower strain accumulation of ferrite phase can further be due to nanocrystalline grains of the ferrite phase.

\section{Conclusion}

The microstructural evolution of cryogenically rolled Ce-SAF2507 was systematically investigated under various levels of deformation conditions.

1. During the cryogenic deformation process, ferrite and austenite grains were squeezed into bands continuously and transformed into the fibrous texture gradually. Many dislocations occur in the high SFE ferrite, which slip and cross-slip to direct plastic deformation. The low SFE austenite mainly coordinates the plastic deformation in the form of twins. At a high deformation level of $90 \%$, the grain size is significantly reduced to the nanoscale.

2. Deformation induces the martensitic transformation of austenite to the martensite phase under the action of stress. Transformation rate increases substantially with the deformation and a rate of $84.1 \%$ is seen at the $90 \%$ deformation level.

3. The hardness of ferrite and austenite was found to increase with the 
increment in the deformation level. Higher work hardening ability of austenite leads to larger hardness increment than that of ferrite. This observation corroborates well with the microstructural evolution and microstrain calculations.

From the above conclusions, the cryogenic rolling has turned to be superior compared to hot and room temperature cold rolling due to ultra-fine grains, higher austenite to martensite transformation, less complicated microstructure evolution during the deformation, and suppressed dynamic recovery in the austenite phase.

\section{Acknowledgment}

Authors acknowledge the financial supports from the National Natural Science Foundation of China with funding Nos. 51801054 and U1804146, Program for Science, Technology Innovation Talents in Universities of Henan Province with No. 17HASTIT026, Education Department of Henan Province with No. 16A430005, as well as Science and Technology Innovation Team of Henan University of Science and Technology with No. 2015XTD006. The project is also financially supported by the Academy of Finland [No. 311934] and Anhui Provincial Grant for high-level platform construction.

\section{References}

[1] H. Sieurin, R. Sandström. Mater. Sci. Eng. A 2006, 418(1-2), 250-256.

[2] F. V. Adams, P. A. Olubambi, J. H. Potgieter, J. Van Der Merwe. Anti-Corros. Method. M. 2010, 57(3), 107-117.

[3] J. O. Nilsson, A. Wilson. Mater. Sci. Tech-Lond. 1993, 9(7), 545-554.

[4] M. K. Mishra, I. Balasundar, A. G. Rao, B. P. Kashyap, N. Prabhu. J. Mater. Eng. Perform. 2017, 26(2), 802-812.

[5] L. Duprez, B. C. De Cooman, N. Akdut. Metall. Mater. Trans. A 2002, 33(7), 1931-1938. 
[6] V. Hosseini, L. Karlsson, S. Wessman, N. Fuertes. Materials 2018, 11(6), 933.

[7] M. Breda, K. Brunelli, F. Grazzi, A. Scherillo, I. Calliari. Metall. Mater. Trans. A 2014, 46(2), 577-586.

[8] T. Zhou, Y. Xiong, Y. Yue, Y. Lu, Y. Chen, T. He, et al. Mater. Sci. Eng. A 2019, $766,138352$.

[9] Y. Xiong, Y. Yue, T. He, Y. Lu, F. Ren, W. Cao. Materials 2018, 11(9), 1557.

[10] A. K. De, D. C. Murdock, M. C. Mataya, et al. Scripta Mater. 2004, 50(12), 1445-1449.

[11] G. K. Williamson, W. H. Hall. Acta Metall. 1953, 1(1), 22-31.

[12] Y. Bi, A. V. Karasev, P. G. Jönsson. ISIJ Int. 2013, 53(12), 2099 - 2109.

[13] J. Ryś, G. Cempura. Mater. Sci. Eng. A 2017, 700, 656-666.

[14] D. Qiu, W. Z. Zhang. Acta Mater. 2007, 55(20), 6754-6764.

[15] L. Chen, F. P. Yuan, P. Jiang, X. L. Wu. Mater. Sci. Eng. A 2012, 551, 154-159.

[16] Z. Gao, J. Li, Y. Wang. Steel Res. Int. 2018, 90(2), 1800397.

[17] H. S. Wang, J. R. Yang, H. K. D. H. Bhadeshia. Mater. Sci. Tech-Lond. 2005, 21(11), 1323-1328.

[18] R. L. Peng, G. C. Chai, T. Stein, T. Manns, S. Johansson. Mater. Sci. Forum 2011, 681, 516-521.

[19] R. Dakhlaoui, C. Braham, A. Baczmański. Mater. Sci. Eng. A 2007, 444(1-2), 6-17.

[20] Q. Ran, W. Xu, Z. Wu, J. Li, Y. Xu, X. Xiao, et al. Metall. Mater. Trans. A 2016, 47(10), 5037-5048.

[21] S. Papula, S. Anttila, J. Talonen, T. Sarikka, I. Virkkunen, H. Hänninen. Mater. Sci. Eng. A 2016, 677, 11-19.

[22] V. Shrinivas, S. K. Varma, L. E. Murr. Metall. Mater. Trans. A 1995, 26(3), 661-671.

[23] H. Liu, B. Liu, P. Bai, H. Sun, D. Li, F. Sun, N. Lin. Mater. Charact. 2015, 107, 262-269.

[24] S. Pramanik, S. Bera, S. K. Ghosh. Steel Res. Int. 2013, 85(5), 776-783.

[25] A. Kumar, R. K. Khatirkar, D. Chalapathi, G. Kumar, S. Suwas. Metall. Mater. Trans. A 2017, 48(5), 2349-2362.

[26] D. Kaoumi, J. Liu. Mater. Sci. Eng. A 2018, 715, 73-82.

[27] S. S. M. Tavares, M. R. da Silva, J. M. Pardal, H. F. G. Abreu, A. M. Gomes. J. Mater. Process. Tech. 2006, 180(1-3), 318-322. 